\title{
Mathematical and Computer Simulation of Pulses Interaction
}

\author{
Yuriy Zayko
}

Department of Applied Informatics, Faculty of Public Administration, the Russian Presidential Academy of National Economy and Public Administration, Saratov Branch, Saratov, Russia

Email address:

zyrnick@rambler.ru

\section{To cite this article:}

Yuriy Zayko. Mathematical and Computer Simulation of Pulses Interaction. American Journal of Mathematical and Computer Modelling. Vol. 3, No. 1, 2018, pp. 22-26. doi: 10.11648/j.ajmcm.20180301.13

Received: February 13, 2018; Accepted: April 14, 2018; Published: May 7, 2018

\begin{abstract}
The paper presents the results of mathematical and computer simulation of the interaction of radio pulses of an arbitrary nature. Pulses of a rectangular and Gaussian shape are considered. We also consider the evolution of the wave packet formed as a result of the decay of the bound quantum state. The main reason for the distortion of the pulse shape is the interaction of narrow-band spectral components of pulses.
\end{abstract}

Keywords: Pulse, Narrowband Component, Fourier-Harmonics, Dispersion, Pulse Distortions

\section{Introduction}

Interference is a typical phenomenon in wave physics. In the propagation of wave impulses, it also takes place, but sometimes its manifestations are very unusual and therefore meet distrust among practitioners. Before analyzing the interference phenomena for pulses, it is necessary to determine clearly what interferes with what. Usually, in experiments on wave interference, there are two waves, which, when superimposed, give an interference pattern. In the case of impulses, the picture is complicated by the fact that the impulse itself is the result of the interference of a large number of its constituent waves-the so-called narrowband components or harmonics. The representation of a pulse in the form of a Fourier integral gives a picture of the distribution of these components over the frequency spectrum. It is necessary to understand the difference between the two. The Fourier harmonic is a narrowband component with an infinitely narrow spectral width and, in general, is a mathematical abstraction. The narrowband component is a physical concept used, for example, in the synthesis of radio frequency filters. The mathematical apparatus that presents a signal and, in particular, an impulse, as a sum of narrow-band components, has not been developed, so we use Fourier analysis. In this article, as well as in the literature in general, these concepts are used as synonyms.

\section{Interference of Classical Impulses}

The initial pulse $E(z=0, t)$, given at the point $z=0$ of the medium, already represents the result of the interference. Subsequently, propagating in a dispersive medium, the impulse is distorted and its distortions are the result of the changed conditions for the combining of its spectral components. Since for most pulses, for example, for rectangular ones, the number of harmonics is large, then the picture of their interaction, i.e. interference picture, looks complicated and confusing. However, it is possible to select the conditions under which a small number of pulse components or pulses will interact, for example, by observing the interaction of two pulses.

Let us consider the problem of the interaction of two pulses propagating in a dispersive medium. The problem of propagation of a single rectangular radio pulse given at the point $z=0$ is described by expressions.

$$
\begin{aligned}
& E(z, t)=\frac{1}{2 \pi} \int_{-\infty}^{\infty} E(\omega) e^{-i \omega t+i h(\omega) z} d \omega \\
& E(\omega)=\int_{-T_{0} / 2}^{T_{0} / 2} E(z=0, t) e^{i \omega t} d t
\end{aligned}
$$

$h(\omega)$ - is the law of dispersion of waves from which a 
pulse is formed, and $T_{0}$ is a pulse duration at the point $z=0$. In the case of an arbitrary dispersion law, this problem has no analytical solution and can only be investigated numerically. For sufficiently long pulses $\left(T_{0} \omega_{0}>>1\right)$ with a good accuracy, one can use the so-called approximation of the dispersion theory when the exact expression for $h(\omega)$ is replaced by its expansion in a Taylor series near the carrier frequency of the pulse filling $\omega_{0}$. For example, for a rectangular pulse in the second approximation of the dispersion theory for $E(z, t)$, was received the expression [1].

$$
E(z, t)=\frac{1-i}{2} \exp \left[i\left(\omega_{0} t-z h_{0}\right)\right]\left\{F^{*}(u)+F^{*}(v)\right\}
$$

where

$$
\begin{aligned}
& u=\frac{\theta}{\sqrt{\pi z\left|h_{0}^{\prime \prime}\right|}}, v=\frac{T_{0}-\theta}{\sqrt{\pi z\left|h_{0}^{\prime \prime}\right|}}, \theta=t-z h_{0}^{\prime}+T_{0} / 2 \\
& h_{0}=h\left(\omega_{0}\right), h_{0}^{\prime}=\frac{d h\left(\omega_{0}\right)}{d \omega}, h_{0}^{\prime \prime}=\frac{d^{2} h\left(\omega_{0}\right)}{d \omega^{2}}
\end{aligned}
$$

and $F$ - is the Fresnel integral:

$$
F(x)=\int_{0}^{x} \exp \left(i \frac{\pi}{2} y^{2}\right) d y
$$

Below the Figure 1 shows the results of calculation by the formula (2) for the case of propagation of two identical rectangular pulses of duration $T_{0}$ generated at the point $z=0$ with a time interval of $2 T_{0}$ and moving in one direction.

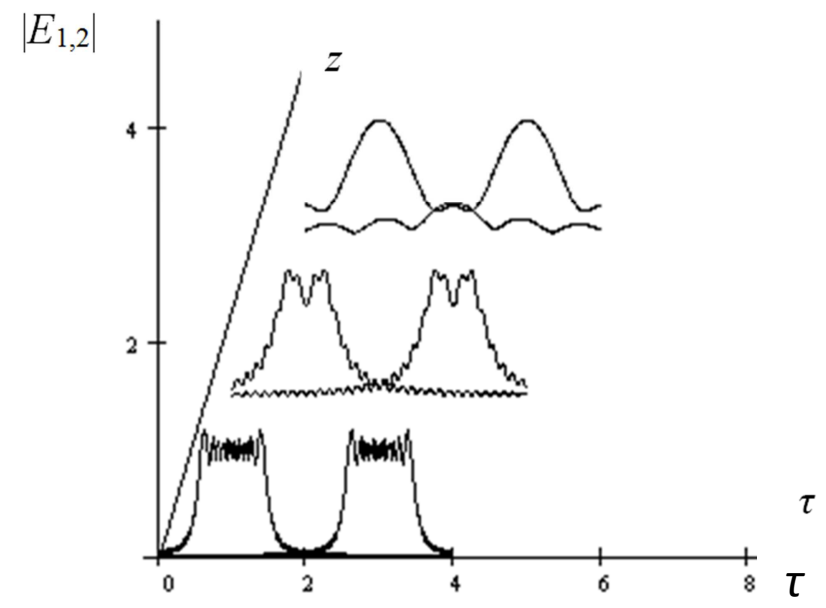

Figure 1. The behavior of the amplitudes $\left|E_{1}\right|$ and $\left|E_{2}\right|$ pulses without taking into account interference phenomena. Pulses are shown for values $N=10$ (lower row), $N=4$ (middle row), $N=1.6$ (upper row). The scale on the $z$-axis is arbitrary, $\tau=\left(t-z h_{0}^{\prime}\right) / T_{0}$. For pulses for different $z$, an arbitrary shift along the $\tau$ axis was applied.

Figure 1 shows the pulse amplitudes $\left|E_{1}(z, t)\right|$ and $\left|E_{2}(z, t)\right|$ at different values of the distance traveled by them. The distortion of the pulses is determined by the parameter $N=\sqrt{d / z}$, where $d=T_{0}^{2} / \pi\left|h_{0}^{\prime \prime}\right|$, which decreases with increasing distance traveled by the impulses. Initial value $N$ $(z=0)=10$, the other two are successively equal 4 and 1.6. When the overlap of pulses is small (small $z, N=10$ ) interference effects can be neglected. But with a significant overlap, this cannot be done and the pattern of amplitude distribution must be calculated by adding the fields $E_{1}(z, t)$ and $E_{2}(z, t)$ of the pulses and only then calculating their total amplitude. The result of this calculation is shown in Figure 2.

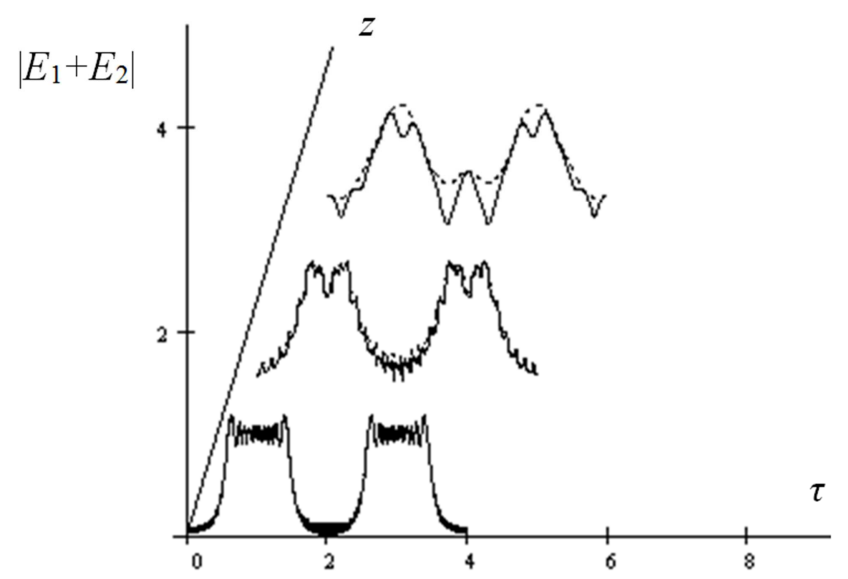

Figure 2. The same as in Figure 1 with allowance for pulse interference (solid lines). Points show the behavior $\left|E_{1}\right|+\left|E_{2}\right|$ for the corresponding pulses.

The amplitude distribution in Figure 2 clearly demonstrates the interference of impulses. Let's pay attention to the fact that even when the impulses do not overlap significantly, their amplitude also contains an oscillating component. There are different opinions about it. From the point of view of practical radio engineering, this is the result of "an essentially different relationship between lowfrequency and high-frequency components in their running spectra" [2]. According to the adherents of the theory of an analytic signal, this is an artifact [3, 4], connected with the shortcomings of the theory of a complex signal (in the terminology of [5]). It was suggested in [4] that these oscillations are also the result of interference of narrow-band components of a signal.

Ideal from the point of view of the theory of an analytic signal is a Gaussian pulse ${ }^{1}$, which, when propagated in a dispersive medium, does not exhibit any amplitude oscillations. From the point of view of interference theory [4], the absence of amplitude oscillations in a Gaussian pulse is due to the fact that it, roughly speaking, represents a single narrow-band component that has nothing to interfere with. At the same time, pulses with a complex envelope, for example, rectangular, contain a large number of narrow-band components, which even in the case of a single pulse give oscillations, interfering with each other.

To prove the interference nature of oscillations, we show that they can also be observed for Gaussian pulses. Consider two Gaussian pulses generated at the point $z=0$, and the

\footnotetext{
${ }^{1}$ Although, as noted in [5], a Gaussian pulse is not an analytical signal, it tends to it when its spectral width is narrowed.
} 
second pulse is delayed relative to the first for a time $T$.

$$
E(0, t)=\exp \left(-i \omega_{0} t\right)\left\{\exp \left(-a t^{2}\right)+\exp \left[-a(t-T)^{2}\right]\right\}
$$

Value $1 / \sqrt{a}<<$ is the half-width of the pulse by level $1 / e$.
Impulses are narrowband, i.e. $\omega_{0} / \sqrt{a}>>1$. The distortion is calculated by formulas (1), in which the second time integral is calculated from $-\infty$ to $+\infty$ since the Gaussian pulse differs from zero anywhere. During spreading, the impulses begin to overlap. In the same notation as above, we obtain.

$$
E(z, t)=\frac{e^{i h_{0} z=i \omega_{0} t}}{\sqrt{1-2 i h_{0}^{\prime \prime} a z}}\left\{\exp \left[\frac{-a\left(t-z h_{0}^{\prime}\right)^{2}}{1-2 i h_{0}^{\prime \prime} a z}\right]+\exp \left[\frac{-a\left(t-T-z h_{0}^{\prime}\right)^{2}}{1-2 i h_{0}^{\prime \prime} a z}\right]\right\}
$$

Using (6), one can obtain an expression for the amplitude $|E(z, t)|$

$$
\begin{aligned}
& |E(z, t)|=(1+\mu)^{-1 / 4}\left\{e^{-2 \delta \sigma^{2}}+e^{-2 \delta(\sigma-T)^{2}}+2 e^{-\delta\left[\sigma^{2}+(\sigma-T)^{2}\right]} \cos \mu \delta\left[\sigma^{2}-(\sigma-T)^{2}\right]\right\}^{1 / 2} \\
& \delta=\frac{a}{1+\mu^{2}}, \mu=2 k_{0}^{\prime \prime} z a, \sigma=t-z k_{0}^{\prime}
\end{aligned}
$$

In Figure 3 is shown the amplitude of the pulses for different values of the parameter $\mu=2 h_{0}^{\prime \prime} z a$. For small $\mu$ the pulses do not overlap and there are no oscillations. For large $\mu$ oscillations of the amplitude arise in the overlap region due to interference. It also follows from (6) that there is no oscillation for one pulse, which agrees with their interference nature.

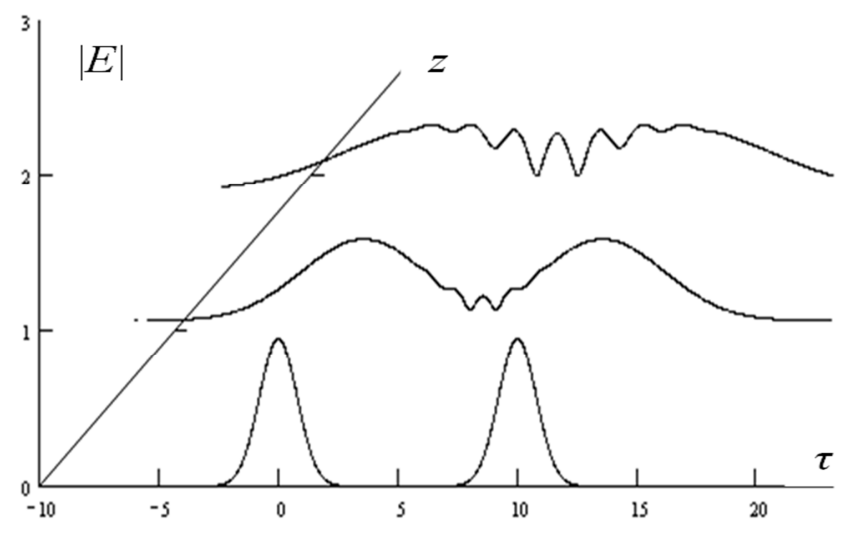

Figure 3. The amplitude of two Gaussian pulses before overlapping (bottom graph, $\mu=0.5)$, for the small overlapping $(\mu=3.5)$ and for the large one (upper graph, $\mu=5.5)$. Pulse delay $\sqrt{a} T=10 ; \tau=\sqrt{a}\left(t-z h_{0}^{\prime}\right)$, $z=\mu / 2 h_{0}^{\prime \prime} a$.

The obtained results, firstly, confirm the interference nature of the distortion of the pulses and, secondly, convince in approximate nature of the Gaussian pulse, often used by the adherents of the theory of analytical signal, which allows it to be used only for rough estimates, but not for calculations ${ }^{2}$.

\section{Distortion of Quantum Packets}

In [4] it was suggested the hypothesis that during the decay of the bound state described by the wave function $\Psi(z, t)$ oscillations of the amplitude $\Psi$ similar to those described above can be observed. This statement requires clarification.

2 The "advantages" of the analytical signal were associated, basically, with extensive practical applications. At the same time, on such "trifles" as the violation of causality simply did not pay attention.
Let's recall the statement of the problem. Wave function $\Psi$ $(z, t)$ of the bound state satisfies the Schrödinger equation with potential $U(z, t)=U(z)$ for $t \leq 0$ and $U(z, t)=0$ for $t>$ 0 . If the potential $U(z)$ is sufficiently smooth, then $\Psi(z, t)$ has no discontinuities and is nonzero everywhere, thus recalling the Gaussian impulse. In this sense, it is the only single narrow-band component, and when freed from the potential $U$, in which it existed before the moment $t=0$, it should not give oscillations. The physical reason for their absence is the absence of conditions for interference.

Consider the problem of the evolution of a wave packet, which until the time $t=0$ represented the wave function of the $n$-th bound state of a harmonic oscillator $A(z, t=0)=\Psi_{n}$ $(z)$, where

$$
\Psi_{n}(z)=\left(\frac{m \omega}{\pi \hbar}\right)^{1 / 4} \frac{1}{\sqrt{2^{n} n !}} \exp \left(-\frac{m \omega}{2 \hbar} z^{2}\right) H_{n}\left(z \sqrt{\frac{m \omega}{\hbar}}\right)
$$

$H_{n}$ - Hermite polynomial, $m, \omega$ - mass and frequency of the oscillator, $\hbar$ - Planck constant [6]. When the potential collapses, the wave packet evolves as the wave function of the free particle in accordance with formulas

$$
\begin{aligned}
& A(z, t)=\frac{1}{2 \pi} \int_{-\infty}^{\infty} A(k) e^{-i \omega(k) t+i k z} d k \\
& A(k)=\int_{-\infty}^{\infty} A(z, 0) e^{-i k z} d z \\
& \omega(k) \approx \omega_{0}+\frac{\hbar k^{2}}{2 m}, \omega_{0}=\frac{E_{0}}{\hbar}, n>>1
\end{aligned}
$$

which are analogous to the expressions (1) for the problem; $E_{0}=\hbar \omega / 2$ - is the energy of the main bound state of the oscillator, $\hbar^{2} k^{2} / 2 m$ is the energy of the free particle, having the wave number $k$. After performing the necessary calculations, we obtain the final expression. 


$$
A(z, t) \approx \frac{\exp \left(-i \omega t / 2+i m z^{2} / 2 \hbar t-i \pi / 4\right)}{\sqrt{2^{n} n !}} \sqrt[4]{\frac{m}{\pi \hbar \omega t^{2}}} H_{n}\left(\sqrt{\frac{m}{\hbar \omega}} \frac{z}{t}\right), \omega t>>1
$$

It is seen from (10) that, for fixed $t$, the pulse, along with the expected spreading, demonstrates an additional spatial quadratic phase modulation. No additional oscillations of the amplitude, like those mentioned above, in accordance with the interference theory, are present.

One can obtain a general formula suitable for investigation the dynamics of a collapsing state for any form of the potential $U(z)$. Combining the first two formulas in (9) we obtain (the value of $\omega_{0}$ is omitted).

$$
\begin{gathered}
A(z, t)=\int A\left(z^{\prime}, 0\right) \Delta\left(z-z^{\prime}, t\right) d z^{\prime} \\
\Delta\left(z-z^{\prime}, t\right)=\sqrt{\frac{m}{2 \pi i \hbar t}} \exp \left[-i \frac{m}{2 \hbar t}\left(z-z^{\prime}\right)^{2}\right] \quad \begin{array}{l}
(4) \text { as in the classical case (see formula (2)). For } \\
\text { the } n-\text { th state in the infinitely deep potentia } \\
A(z, 0)=\sqrt{2 / a} \sin n \pi z / a \text { [6], where } a \\
\text { thickness, from (11) one can obtain, to within a } \\
\text { phase factor. }
\end{array} \\
A(z, t) \sim \frac{1}{\sqrt{2 a}}\left\{e^{i(x+\Delta x)}\left[F^{*}(x+\Delta x)+F^{*}(b-x-\Delta x)\right]-e^{i(x-\Delta x}\left[F^{*}(x-\Delta x)+F^{*}(b-x+\Delta x)\right]\right\} \\
x=\sqrt{\frac{m}{\pi \hbar t}} z, b=\sqrt{\frac{m}{\pi \hbar t}} a, \Delta x=\frac{2 n}{b}
\end{gathered}
$$

$F(z)$ - Fresnel integral (4). Calculations show that in this case, the same amplitude oscillations appear as in the classical case.

\section{Conclusions}

Based on the results of the article and previous works [716] it can be argued that the presence or absence of oscillations of the quadrature components of the signal (pulse) -the amplitude and the instantaneous frequency is due to the existence of narrow-band signal components and interference between them. The situation is the same for both classical and quantum physics. The connection between the narrow-band signal components and the spectral components of the Fourier spectrum continues to be interesting and requires further investigation.

\section{References}

[1] Ginzburg V. L., Electromagnetic Waves Propagation in Plasma. 1964. (N. Y.: Pergamon Press).

[2] Verbin Yu. P., On the estimation of the speed of propagation of a radio signal//Radiotechnica i Electronica .-1995.- V. 40, № 8, p. 1169-1176,(Russian).

[3] Vakman D. E., On fast oscillations of the parameters of oscillations// Radiotechnica i Electronica.-1986, V. 31, № 7, p. 1459-1462,(Russian).

[4] Zayko Yu. N., The history of one "artifact"// Proc. of the Saratov State University, ser. Physics, 2012, V. 12, № 1, p. 3 12, (Russian).
The integration in (11) is performed over the domain in which $A(z, 0)$ is different from zero. Function $\Delta\left(z-z^{\prime}, t\right)$ satisfies the condition $\Delta\left(z-z^{\prime}, t\right) \underset{t \rightarrow 0}{\longrightarrow} \delta\left(z-z^{\prime}\right)$, where $\delta$ $\left(z-z^{\prime}\right)$ - is the Dirac delta-function. Formula (11) can be applied to solve the problem in the case of a potential $U(z)$ that does not satisfy the smoothness condition, for example, for a potential in the form of a rectangular potential well with infinite walls. It is easy to show that the solution, in this case, can be expressed with the help of the same Fresnel integrals (4) as in the classical case (see formula (2)). For example, for the $n-$ th state in the infinitely deep potential well, when $A(z, 0)=\sqrt{2 / a} \sin n \pi z / a$ [6], where $a$ - is the well's thickness, from (11) one can obtain, to within an unimportant
[5] Vainshtein L. A., Vakman D. E., Amplitude, phase, frequency the basic concepts of the theory of oscillations//Uspehi Fizicheskih Nauk, 1977, V. 123, №. 4, p. 657-682, (Russian).

[6] Landau L. D. \& Lifshitz E. M., Quantum Mechanics (Volume 3 of A Course of Theoretical Physics ) Pergamon Press, 1965.

[7] Johler J. T., The Propagation Time of a Radio Pulse// IEEE Trans., 1963, Vol. AP-11, P. 661-668.

[8] Strelkov G. M., Propagation of a Radio Pulse in Isotropic Plasma// Radiotechnica i Electronica.-2006, V. 51, p. 672-682, (Russian).

[9] Srtelkov G. M., Complex Radio Signal in Ionospheric Plasma// Radiotechnica i Electronica.-2008, V. 53, № 1, p. 1094-1103, (Russian).

[10] Srtelkov G. M., Naryshkin V. I., Propagation of a Radio Pulse with Linear Frequency Modulation in Isotropic Plasma// Radiotechnica i Electronica.-2008, V. 53, № 1, p. 49-57, (Russian).

[11] Shvartsburg A. B., Impulse electrodynamics of nonharmonic signals//Uspehi Fizicheskih Nauk, 1994, V. 164, № 3, P. 333335 (Russian).

[12] Shvartsburg A. B., Video Pulses and Non-Periodic Waves in Dispersive Media (exactly solvable models)//Uspehi Fizicheskih Nauk, 1998, V. 168, № 1, P. 85-103 (Russian).

[13] Zayko Yu. N., Frequency Modulation of the Filling of a Radio Pulse Propagating in a Waveguide//Izvestia Vyzov: Radiofizika, 1989, V. 32, № 12, P. 1558-1560 (Russian).

[14] Zayko Yu. N., Mezhuev D. I., Propagation of a Rectangular Radio Pulse with Linear Frequency Modulation in a Dispersive Medium// Technical Physics Letters, 1991, V. 17, № 17, P. 50-53. 
[15] Zayko Yu. N., The Geometrical Phase of Modulated Waves, Propagating in Dispersive Media//Applied Math. Letters, 1997, V. 10, № 5, P. 75-78.
[16] Zayko Yu. N., Interaction of Narrow-Band Signal Components and Complex Behavior of PDE Characteristics in $\mathrm{x}, \mathrm{t}-$ plane//Proc SPIE, 2006, V. 6165, P. i06. 